\title{
Oltipraz Prevents High Glucose-Induced Oxidative Stress and Apoptosis in RSC96 Cells through the Nrf2/NQO1 Signalling Pathway
}

\author{
Zengxin Jiang, ${ }^{1,2}$ Mengxuan Bian, ${ }^{1}$ Jingping Wu, ${ }^{1}$ Defang $\mathrm{Li}^{1}{ }^{1}$ Lei Ding, \\ and Qingmin Zeng $\mathbb{D}^{1}$
}

${ }^{1}$ Department of Orthopedic Surgery, Fudan University Jinshan Hospital, Longhang Road, Jinshan, Shanghai 201508, China

${ }^{2}$ Department of Orthopedic Surgery, Zhongshan Hospital, Fudan University, Shanghai 200032, China

Correspondence should be addressed to Qingmin Zeng; qingminzeng1703@163.com

Received 12 March 2020; Accepted 6 June 2020; Published 24 June 2020

Academic Editor: Yunfeng Zhao

Copyright ( 2020 Zengxin Jiang et al. This is an open access article distributed under the Creative Commons Attribution License, which permits unrestricted use, distribution, and reproduction in any medium, provided the original work is properly cited.

\begin{abstract}
Diabetic peripheral neuropathy (DPN) is a common complication of diabetes mellitus (DM). Schwann cell (SC) apoptosis contributes to the occurrence and development of DPN. Effective drugs to prevent SC apoptosis are required to relieve and reverse peripheral nerve injury caused by DM. Oltipraz [4-methyl-5-(2-pyrazinyl)-1,2-dithiole-3-thione], an agonist of nuclear factor erythroid derived-2-related factor 2 (Nrf2), exerts strong effect against oxidative stress in animal models or clinical patients in certain diseases, including heart failure, acute kidney injury, and liver injury. The aim of the present study was to determine the effectiveness of oltipraz in preventing SC apoptosis induced by high glucose levels. RSC96 cells pretreated with oltipraz were cultured in high-glucose medium $(50 \mathrm{mM}$ glucose) for $24 \mathrm{~h}$, and cells cultured in medium containing $5 \mathrm{mM}$ glucose were used as the control. Flow cytometry was used to evaluate the degree of apoptosis. A Cell Counting Kit- 8 assay was used to assess cell viability. The mitochondrial membrane potential was assessed using JC-1 staining, and reactive oxygen species (ROS) generation was measured using 20,70-dichlorodihydrofluorescein diacetate staining. In addition, the levels of malondialdehyde (MDA) and superoxide dismutase (SOD) levels were also evaluated using the corresponding kits. Flow cytometry was subsequently used to detect apoptosis, and western blotting was used to measure the expression levels of nuclear factor erythroid derived-2-related factor 2 and NADPH quinone oxidoreductase 1. The results showed that high glucose concentration increased oxidative stress and apoptosis in RSC96 cells. Oltipraz improved cell viability and reduced apoptosis of RSC96 cells in the high glucose environment. Additionally, oltipraz exhibited a significant antioxidative effect, as shown by the decrease in MDA levels, increased SOD levels, and reduced ROS generation in RSC96 cells. The results of the present study suggest that oltipraz exhibits potential as an effective drug for treatment with DPN.
\end{abstract}

\section{Introduction}

Diabetes mellitus (DM) is a systemic metabolic disease characterized by high blood glucose levels. DM is the most common cause of neuropathy worldwide, and up to $50 \%$ of all patients with DM may develop neuropathy [1-3]. Diabetic peripheral neuropathy (DPN) is a common complication of $\mathrm{DM} ; 50 \%$ of the patients with DPN are asymptomatic, whereas others may suffer from complicated symptoms such as pain, foot ulcers, and paresthesia $[4,5]$. DPN severely affects patients' quality of life and thus presents a significant economic burden to patients.

Schwann cells (SCs) are the most common type of glia in peripheral nerves. SCs ensheath all the axons of the peripheral nerves and secrete neurotrophic factors, which help maintain the structure and function of peripheral nerves $[6,7]$. Increasing evidence has shown that SCs serve an important role in DPN [8-13]. SC apoptosis induced by a high glucose environment is thought to be one of the primary causes of DPN. Increased SC apoptosis was observed in 
diabetic $(\mathrm{db} / \mathrm{db})$ mice and diabetic rats treated with streptozotocin [12-15]. A previous study showed that inhibiting SC apoptosis could alleviate myelin sheath injury and delay peripheral nerve degeneration in DPN [15-17].

SCs are hypothesized to be an important site of reactive oxygen species (ROS) generation in peripheral nerves [10]. Hyperglycaemia promotes excessive ROS production in SCs primarily through the polyol (sorbitol) pathway, the mitochondrial electron transport chain, increased production of advanced glycation end products, NADPH oxidases, and nitric oxide synthases [18-20]. Excessive ROS levels induced by hyperglycaemia in SCs can increase intracellular oxidative stress which results in cell apoptosis, promoting the pathological mechanisms underlying peripheral neuropathy $[21,22]$. Preventing oxidative stress in SCs may thus be a suitable method for preventing apoptosis of SCs.

Oltipraz [4-methyl-5-(2-pyrazinyl)-1,2-dithiole-3-thione] is an agonist of nuclear factor erythroid derived-2-related factor 2 (Nrf2), an important transcription factor involved in regulating the intracellular antioxidant response [23, 24]. Nrf2 functions as an activator of antioxidant response element (ARE), which is recognized as the cis-element essential for the basal and inducible expression of several antioxidant genes, including NADPH quinone oxidoreductase 1 (NQO-1) [25-27]. Oltipraz and its oxidized metabolites have proven strong antioxidant effects in animal models or clinical patients in certain diseases [28-32]. However, it is still unclear whether oltipraz may reduce apoptosis of SCs through reducing oxidative stress. The aim of the present study was to investigate the effect of oltipraz on apoptosis of SCs induced by high glucose.

\section{Materials and Methods}

2.1. Cell Culture and Treatment. RSC96 cells, a rat SC line, was purchased from The Cell Bank of Type Culture Collection of the Chinese Academy of Sciences. RSC96 cells were cultured in DMEM (Gibco; Thermo Fisher Scientific, Inc.) supplemented with 10\% FBS (Thermo Fisher Scientific, Inc.) with $5 \% \mathrm{CO}_{2}$ at $37^{\circ} \mathrm{C}$. Cells were trypsinized and subcultured in 6 -well plates $\left(5 \times 10^{5}\right.$ cells/well) or 96 -well plates $\left(1 \times 10^{4}\right.$ cells/well $)$ for subsequent experiments. After reaching $\sim 70 \%$ confluence, RSC96 cells were cultured in DMEM supplemented with $10 \%$ FBS ( $5 \mathrm{mM}$ glucose; control) or high-glucose DMEM supplemented with 10\% FBS (50 mM glucose) for $24 \mathrm{~h}$. Cells were pretreated with $20 \mu \mathrm{M}$ oltipraz (Sigma-Aldrich; Merck KGaA) for $24 \mathrm{~h}$ prior to high glucose treatment. For the control conditions, $45 \mu \mathrm{M}$ mannitol was used to match the hyperosmolality of the cells cultured in the hyperglycemic conditions. The glucose concentrations and oltipraz concentrations used in the present study were based on previous studies $[15,31]$.

2.2. Determination of Cell Viability. The viability of RSC96 cells was assessed using a Cell Counting Kit-8 (CCK-8) assay (KeyGen Biotech). Briefly, cells were subcultured in 96-well plates $\left(1 \times 10^{4}\right.$ cells/well). Following high glucose treatment for $24 \mathrm{~h}$, the cells were incubated with $10 \mu \mathrm{l} \mathrm{CCK-8} \mathrm{solution}$ combined with $100 \mu \mathrm{l}$ serum-free DMEM at $37^{\circ} \mathrm{C}$ for $2 \mathrm{~h}$.

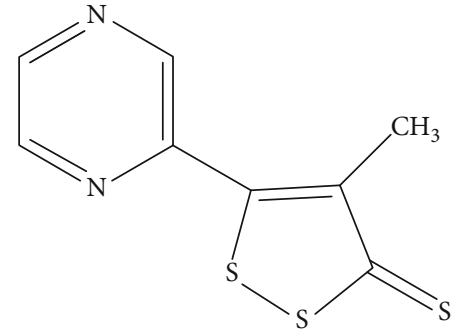

FIGURE 1: The structural formula of oltipraz.

The absorbance at $450 \mathrm{~nm}$ was measured using a microplate reader (Epoch; BioTek Instruments, Inc.).

2.3. Determination of Mitochondrial Membrane Potential. JC-1 staining (Beyotime Institute of Biotechnology) was used to evaluate the mitochondrial membrane potential of RSC96 cells. Cells were washed twice with PBS (KeyGen Biotech) and incubated with JC-1 working buffer at $37^{\circ} \mathrm{C}$ for $20 \mathrm{~min}$. The cells were then washed twice with JC-1 staining buffer and observed under a fluorescence microscope (magnification, $\mathrm{x} 100)$. In addition, the mitochondrial membrane potential of RSC96 cells was analysed using a BD Accuri C6 Plus flow cytometer (BD Biosciences) and FlowJo software (version 10.0.7). Green fluorescence was detected in the FL1 channel, and red fluorescence was detected in the FL2 channel.

2.4. Determination of Cell Apoptosis. RSC96 cell apoptosis was assessed using Annexin V-fluorescein isothiocyanate (FITC)/propidium iodide (PI) staining (BD Biosciences). RSC96 cells were digested with EDTA-free trypsin (Gibco; Thermo Fisher Scientific, Inc.). Cells were washed twice with ice-cold PBS and resuspended in $100 \mu$ l binding buffer. Cells were incubated with $5 \mu \mathrm{l}$ Annexin V-FITC and $5 \mu \mathrm{l}$ PI for $15 \mathrm{~min}$ at room temperature in the dark. Apoptosis was analysed using flow cytometry within $30 \mathrm{~min}$ of staining.

2.5. Determination of Intracellular ROS. 20,70-Dichlorodihydrofluorescein diacetate (DCFH-DA; Sigma-Aldrich; Merck $\mathrm{KGaA}$ ) was used to assess intracellular ROS levels in RSC96 cells. Cells were collected and resuspended in $10 \mathrm{mM} \mathrm{DCFH-}$ DA solution with serum-free DMEM and incubated at $37^{\circ} \mathrm{C}$ for $20 \mathrm{~min}$. The cells were then washed three times with serum-free DMEM to remove extracellular DCFH-DA. Flow cytometry was used to detect ROS levels with an excitation wavelength of $488 \mathrm{~nm}$ and an emission wavelength of $519 \mathrm{~nm}$.

2.6. Determination of Malondialdehyde (MDA) and Superoxide Dismutase (SOD) Levels. A lipid peroxidation MDA assay kit (Beyotime Institute of Biotechnology) was used to measure MDA levels, and a WST-8 assay kit (Beyotime Institute of Biotechnology) was used to detect SOD levels according to the manufacturer's protocol. Briefly, cell lysates were incubated with working solution for $30 \mathrm{~min}$ at $37^{\circ} \mathrm{C}$. Absorbance was measured at $450 \mathrm{~nm}$ for SOD and at $523 \mathrm{~nm}$ for MDA using a microplate reader. Total protein concentration was measured using a bicinchoninic acid (BCA) protein assay kit (Thermo Fisher Scientific, Inc.) and was used to normalize the MDA and SOD levels. 


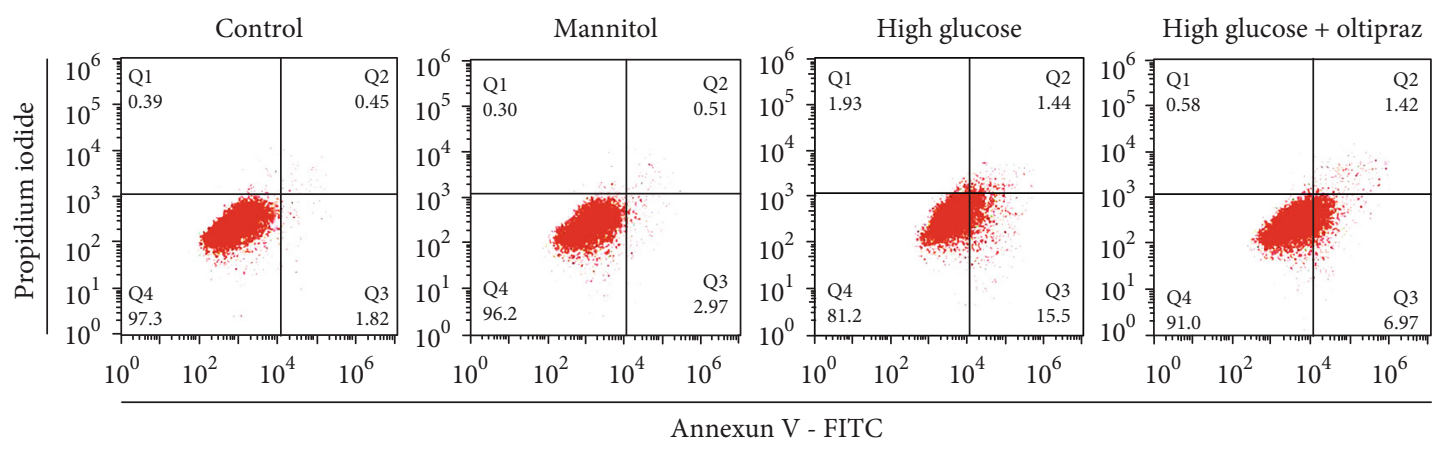

(a)

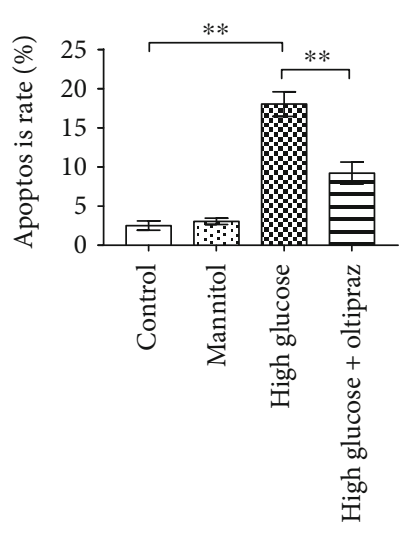

(b)

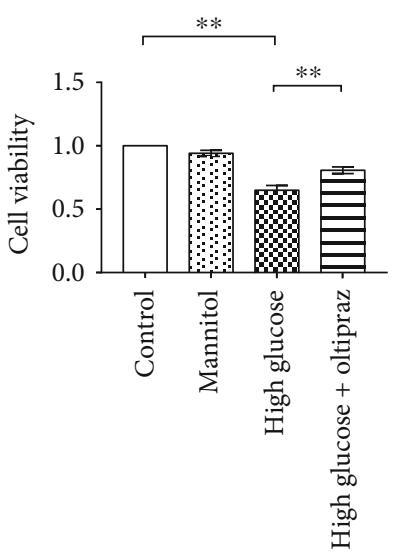

(c)

FIgURe 2: Flow cytometry was used to detect apoptosis of RSC96 cells. (a) Representative flow cytometry histograms. (b) Apoptotic rate of RSC96 cells in the different groups. The apoptotic rate in the high-glucose treatment group was significantly increased compared with the control group. Oltipraz reduced the apoptotic rate compared with high-glucose treatment alone. (c) Cell viability in the different groups after $24 \mathrm{~h}$ of treatment. Significant differences were observed among all groups in apoptosis rate and cell viability $(P<0.01$, one-way ANOVA). ${ }^{* *} P<0.01$.

2.7. Western Blot Assay. Western blotting was used to evaluate the expression levels of Nrf2 and NQO1. After $24 \mathrm{~h}$ of treatment, total protein was extracted from RSC96 cells using radioimmunoprecipitation assay buffer (Sigma-Aldrich; Merck KGaA). The samples were sonicated for $10 \mathrm{sec}$. Following centrifugation at 2,000 $\times \mathrm{g}$ for $15 \mathrm{~min}$ at $4^{\circ} \mathrm{C}$, protein concentrations were measured using a BCA protein assay kit. Total protein $(20 \mu \mathrm{g}$ per sample) was separated by SDSPAGE on a $12 \%$ gel and subsequently transferred to a nitrocellulose membrane (EMD Millipore). The membranes were incubated overnight at $4^{\circ} \mathrm{C}$ with the following antibodies: Anti-Nrf2 (1:1,000; cat. no. 12721; Cell Signalling Technology Inc.), anti-NQO1 (1:1,000; cat. no. ab80588; Abcam), or anti$\beta$-actin $(1: 1,000$; cat. no. 4970; Cell Signalling Technology, Inc.). Membranes were washed with TSB-Tween (0.05\%) for 30 min and incubated with an anti-rabbit secondary antibody (1:5,000; cat. no. 7074; Cell Signaling Technology, Inc.) for $2 \mathrm{~h}$ at room temperature. Enhanced chemiluminescence Plus (Tanon Science and Technology Co., Ltd.) was used to visualize the protein bands. Densitometry analysis was performed using ImageJ software (version 1.8.0; National Institutes of Health).

2.8. Statistical Analysis. Each experiment was independently performed at least three times. Data are expressed as the mean \pm standard deviation. Statistical analysis was performed using SPSS software (version 20.0; IBM Corp.). Statistical comparisons among different groups were performed using one-way ANOVA followed by Bonferroni's multiple comparison test. $P<0.05$ was considered to indicate a statistically significant difference.

\section{Results}

3.1. Oltipraz Ameliorates High Glucose Induced RSC96 Cell Injury. The structural formula of oltipraz was shown in Figure 1. A CCK8 assay was used to determine the viability of RSC96 cells. The results showed that $50 \mathrm{mM}$ high glucose reduced the viability of RSC96 cells $(P<0.01)$, and treatment with Oltipraz significantly increased cell viability in response to high glucose conditions $(P<0.01)$ (Figure $2(\mathrm{c})$ ).

Depolarized mitochondrial membrane potential is considered a primary marker of early cell apoptosis. JC-1 staining was used to evaluate the mitochondrial membrane potential of RSC96 cells. The control group showed red fluorescence whereas the cells incubated in high-glucose showed increased green fluorescence when observed under the fluorescence microscope, suggesting that high-glucose conditions depolarized the mitochondrial membrane potential in RSC96 cells. Green fluorescence was decreased in the oltipraz-treated cells compared with the cells in the high- 

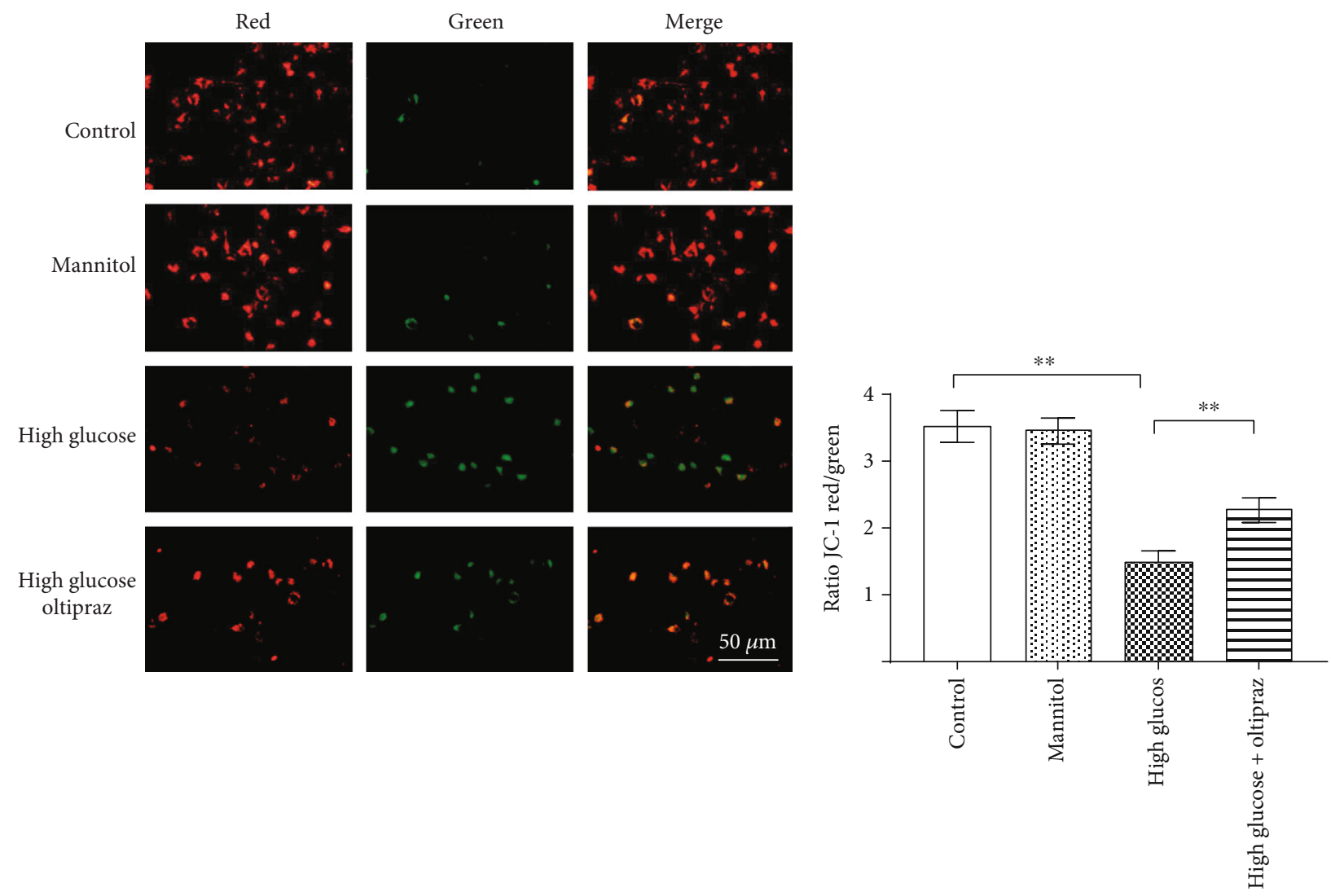

(a)

(b)

FIGURE 3: JC-1 staining was used to detect cell apoptosis. (a) Fluorescence microscopy showed that the mitochondrial membrane potential of RSC96 cells was decreased (green fluorescence) in the high-glucose group compared with the control group. Oltipraz prevented mitochondrial damage as a result of treatment with high glucose. (b) Flow cytometry analysis showed that the red/green fluorescence ratio of cells in the high-glucose group was higher compared with the control group. Oltipraz treatment significantly increased the red/green fluorescence ratio compared with the high-glucose group. Scale bar, $50 \mu \mathrm{m}$. Significant differences were observed among all groups $\left(P<0.01\right.$, one-way ANOVA). ${ }^{* *} P<0.01$.

glucose group (Figure 3(a)). Flow cytometry analysis also showed that the red/green fluorescence ratio in the oltipraztreated cells was higher compared with the high-glucose group $(P<0.01)$, suggesting that oltipraz protected RSC96 cells against glucose-induced mitochondrial damage (Figure 3(b)).

Annexin V/PI staining was subsequently used to investigate RSC96 cell apoptosis (Figure 2(a)). Flow cytometry analysis showed that the apoptotic rate in the high-glucose group was significantly increased compared with the control group $(P<0.01)$, and oltipraz treatment significantly reduced apoptosis compared with the high-glucose group $(P<0.01)$, indicating that oltipraz protected against apoptosis induced by high glucose in RSC96 cells (Figure 2(b)). Mannitol was used to normalize the hyperosmolality, and the results showed that hyperosmolality did not affect cell viability or apoptosis.

3.2. Oltipraz Inhibits ROS Generation. After $24 \mathrm{~h}$ of treatment, DCFH-DA staining was used to measure the ROS levels of RSC96 cells in different groups (Figure 4(a)). Flow cytometry analysis showed that the proportion of positively stained cells in the high-glucose group was significantly higher compared with the control group $(P<0.01)$. Oltipraz treatment reduced the proportion of positively stain cells in the high glucose-treated cells compared with high-glucose treatment alone $(P<0.01)$. Mannitol did not affect ROS production (Figure 4(b)). The results demonstrated that oltipraz effectively prevented excessive ROS generation as a result of the high glucose conditions.

3.3. Oltipraz Increases SOD Levels and Decreases MDA Levels. A lipid peroxidation MDA assay kit was used to measure MDA levels and a WST-8 assay was used to assess SOD levels. The results showed that high glucose conditions increased MDA levels and decreased SOD levels in RSC96 cells (Figures 4(c) and 4(d); $P<0.01$ ). Compared with highglucose treatment alone, oltipraz treatment significantly reduced MDA levels and increased SOD levels $(P<0.01)$. There was no significant difference between the mannitol group and the control group. The lower levels of MDA and higher levels of SOD in the oltipraz-treated cells suggesting that oltipraz protected RSC96 cells from oxidative stress.

3.4. Oltipraz Treatment Increases the Expression of Nrf2 and NQO1. Western blotting was used to measure the expression levels of Nrf2 and NQO1 in RSC96 cells in the four groups. Western blot analysis showed that in the oltipraz groups, 

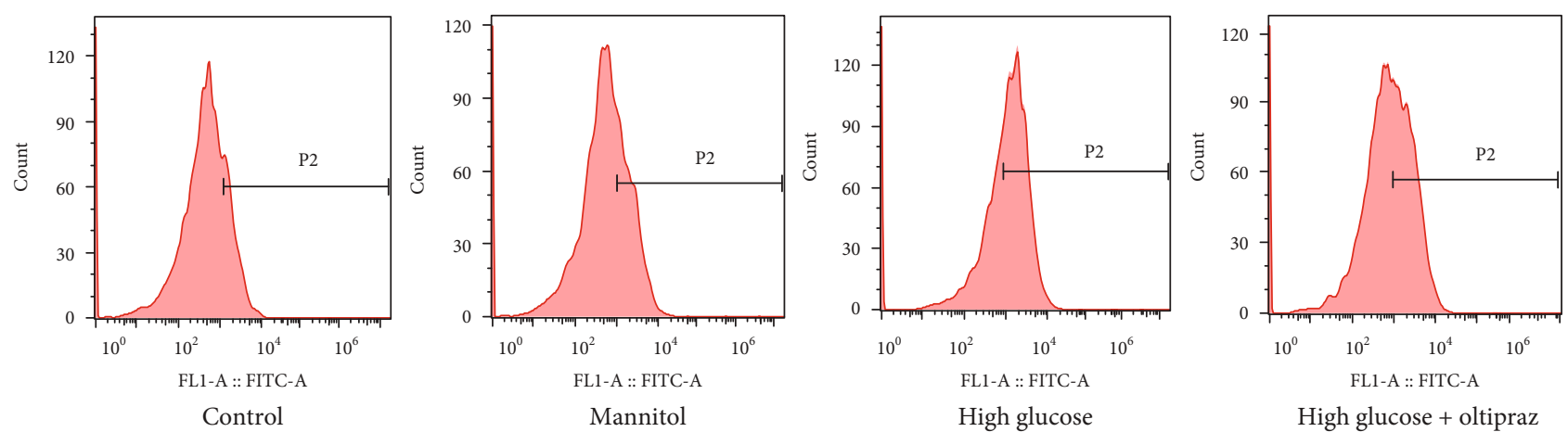

(a)

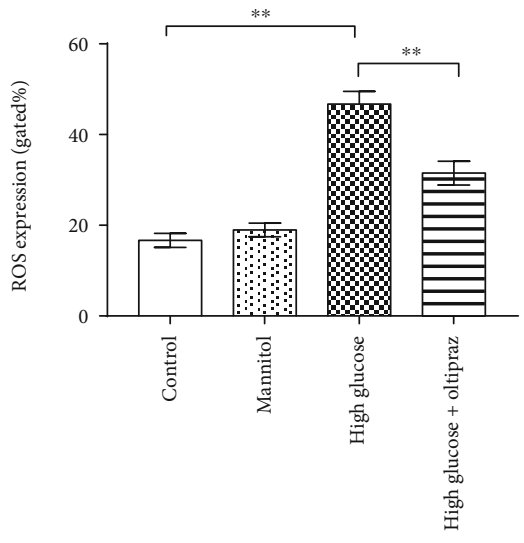

(b)

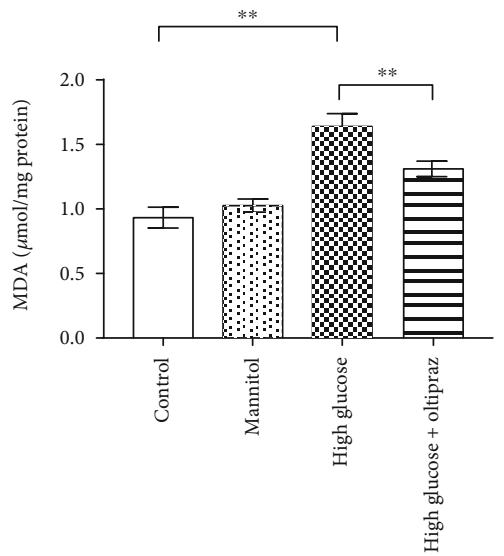

(c)

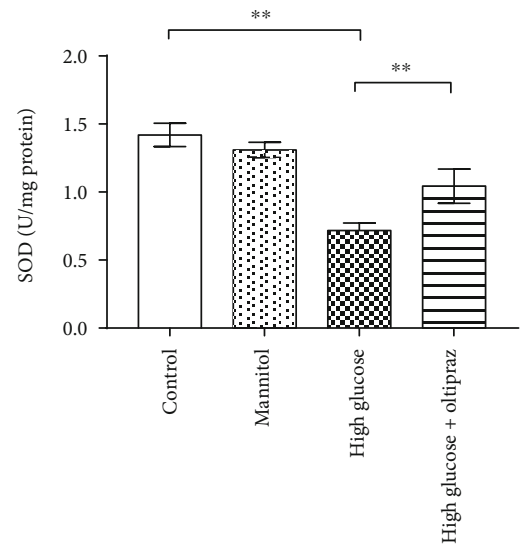

(d)

FIgURE 4: DCFH-DA staining was used to detect RSC96 cell apoptosis. (a) Representative flow cytometry histograms. (b) Flow cytometry analysis showed that high glucose levels significantly increased ROS generation, and oltipraz treatment reduced excessive ROS generation. (c) MDA levels of RSC96 cells in the different groups. (d) SOD levels of RSC96 cells in the different groups. Significant differences were observed among all groups in levels of ROS, MDA, and SOD $\left(P<0.01\right.$, one-way ANOVA). ${ }^{* *} P<0.01$. DCFH-DA: 20,70dichlorodihydrofluorescein diacetate.

the expression levels of Nrf2 and NQO1 were significantly increased compared with the high glucose group $(P<0.01)$. There was no significant difference observed between the mannitol group and the control group (Figure 5). The results show that oltipraz treatment significantly increased the expression of Nrf2 and the downstream molecule NQO1, and thus exerted a potential antioxidant effect.

\section{Discussion}

Previous studies have demonstrated that apoptosis of SCs induced by high glucose contributes to the development of DPN [12-15]. Effective drugs to prevent apoptosis of SCs are important for relieving and reversing peripheral nerve injury caused by DM. The present study showed that oltipraz effectively prevented oxidative stress caused by high glucose, and thus reduced SC apoptosis. Oltipraz treatment decreased MDA levels, increased SOD levels, reversed excessive ROS generation in SCs incubated with high glucose, and protected SCs from mitochondrial damage. Oltipraz treatment increased the expression of Nrf2 and the downstream molecule NQO1 in SCs incubated in a high-glucose environment, and this may underlie the beneficial antioxidant effects of oltipraz.
Peripheral neuropathy is the most common complication of diabetes, with a prevalence of up to $50 \%$ in diabetic patients [2-5]. Several drugs have been reported to be less effective, such as oxcarbazepine and acetyl L-carnitine [33-35]. Therefore, we still need to find more effective treatments. Although the pathogenesis of DPN is not fully understood, increasing evidence has shown that SC injury is one of the characteristics of DPN [8-13]. Oxidative stress induced by high glucose conditions in DM is a primary cause of tissue damage. SCs are considered an important producer of ROS. Excessive ROS levels caused by high glucose levels result in mitochondrial damage and apoptosis in SCs, affecting the protective effect and nerve repair function of SCs [9]. In addition, SC interactions with other tissues, including axons and microvessels are disrupted, which contribute to the occurrence and development of DPN [10]. Therefore, preventing oxidative stress in SCs in a high glucose environment, and further preventing the excessive apoptosis of SCs, may be an effective method for treating DPN.

Nrf2 is a transcription factor that controls the basal and induced expression of a range of antioxidant enzymes. The Nrf2 signalling system involves interacting proteins and regulatory molecules to counter oxidative stress. Studies have reported that Nrf2 has an antioxidant effect through 


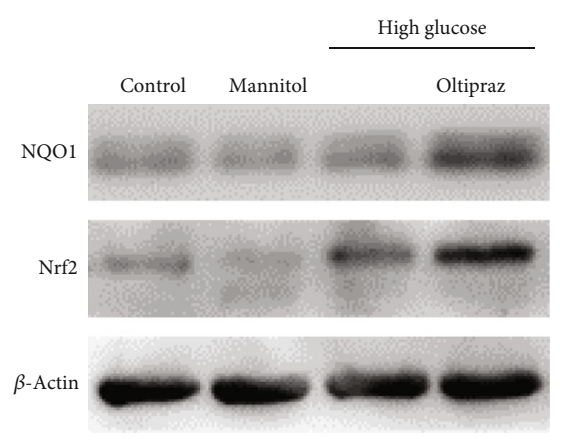

(a)

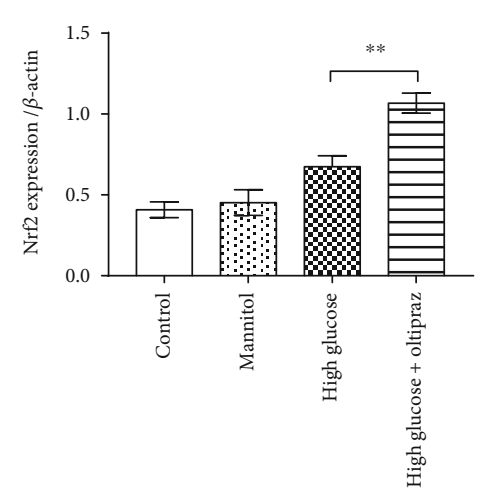

(b)

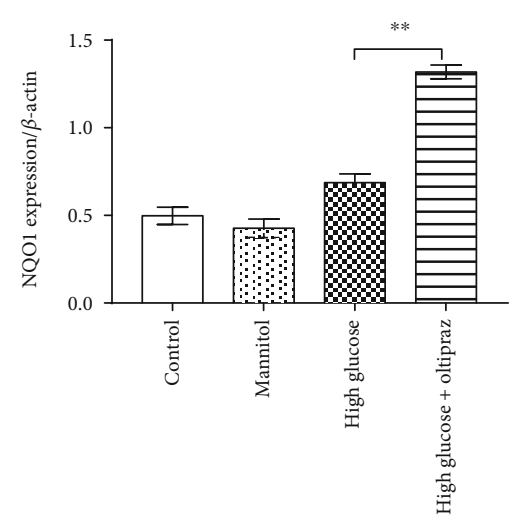

FIgURE 5: Western blotting was used to assess the expression levels of Nrf2 and NQO1. (a) Representative western blotting image showing Nrf2 and NQO1 expressions in SCs. (b) Oltipraz treatment significantly increased the expression levels of Nrf2 and NQO1 compared with cells incubated in high-glucose conditions without treatment. Significant differences were observed among all groups in Nrf2 and NQO1 expression $\left(P<0.01\right.$, one-way ANOVA). ${ }^{* *} P<0.01$. Nrf2: nuclear factor erythroid derived-2-related factor 2; NQO-1: NADPH quinone oxidoreductase 1.

inducing expression of the NQO1 isoenzyme $[25,26]$. Oltipraz activates Nrf2 and subsequently increases the expression of genes encoding antioxidants. Oltipraz has been shown to be effective in animal models of certain diseases due to its antioxidant properties, including heart failure, acute kidney injury, and liver injury [30-32]. Therefore, oltipraz may potentially inhibit SC apoptosis by preventing oxidative stress.

The present study demonstrated that high glucoseinduced oxidative stress promoted RSC96 cell apoptosis. To mimic hyperglycemia, $50 \mathrm{mM}$ glucose was used in the highglucose group, and $5 \mathrm{mM}$ glucose was used as the control. Mannitol was used to normalize the hyperosmolality of hyperglycemia. The results showed that high-glucose levels promoted ROS generation, increased MDA levels, and decreased SOD levels in SCs, suggesting that high-glucose conditions induced oxidative stress in SCs. Furthermore, incubation with high glucose depolarized the mitochondrial membrane potential, suggesting the presence of mitochondrial damage. Oxidative stress results in SC injury, consistent with the reduced viability and increased apoptosis of SCs treated with high-glucose in the present study. Oltipraz treatment exhibited a strong antioxidant capacity with low ROS levels, low MDA levels, and high SOD levels. Mitochondria are the primary source and target of ROS. Mitochondria are damaged as a result of oxidative stress, and mitochondrial damage is used as a marker of cell apoptosis and oxidative stress [36]. Oltipraz treatment alleviated mitochondrial damage, and thus improved cell viability and prevented cell apoptosis. Western blotting demonstrated that oltipraz increased Nrf2 and NQO1 protein expression in SCs. Thus, it is hypothesized that oltipraz prevented oxidative stress through the activation of the Nrf2/NQO1 signalling pathway.

In conclusion, the present study showed that oltipraz effectively prevents oxidative stress induced by high glucose, and thus reduced SC apoptosis through increasing the expression of Nrf2 and its downstream signalling molecule, NQO1. However, considering that SCs are not the only type of cells damaged in DPN, the effectiveness of oltipraz for the treatment of other types of damaged neural tissues and cells should be examined. In addition, further experiments in vivo are required to determine whether oltipraz may effectively relieve the symptoms of DPN. However, the present study highlights the potential of oltipraz for the treatment of DPN.
Abbreviations
DM: Diabetes mellitus
DPN: Diabetic peripheral neuropathy
SC: $\quad$ Schwann cell
ROS: $\quad$ Reactive oxygen species
MAPK: Mitogen-activated protein kinase
Nrf2: $\quad$ Nuclear factor erythroid derived-2-related factor 2 NQO-1: NADPH quinone oxidoreductase 1.

\section{Data Availability}

The data used to support the findings of this study are available from the corresponding author upon request.

\section{Conflicts of Interest}

The authors declare that they have no competing interests.

\section{Authors' Contributions}

QZ and JW designed the study. ZJ, MB, and LD conducted the majority of the experiments. ZJ and DL assisted with the analysis and interpretation of data. ZJ and MB prepared the manuscript. All authors were involved in drafting the 
article or revising it critically for important scientific content. Zengxin Jiang and Mengxuan Bian as first authors have contributed equally to this work.

\section{Acknowledgments}

The present study was supported by the Shanghai Jinshan Municipality Health Bureau (grant no. JSKJ-KTMS-2018-01) and the Shanghai Jinshan District Science and Technology Commission (grant no.2019-3-03).

\section{References}

[1] C. A. Abbott, R. A. Malik, E. R. E. van Ross, J. Kulkarni, and A. J. M. Boulton, "Prevalence and Characteristics of Painful Diabetic Neuropathy in a Large Community-Based Diabetic Population in the U.K.," Diabetes Care, vol. 34, no. 10, pp. 2220-2224, 2011.

[2] A. M. Vincent and E. L. Feldman, "New Insights into the Mechanisms of Diabetic Neuropathy," Reviews in Endocrine and Metabolic Disorders, vol. 5, no. 3, pp. 227-236, 2004.

[3] J. Pirart, "Diabetes mellitus and its degenerative complications: a prospective study of 4, 400 patients observed between 1947 and 1973 (author's transl)," Diabete Metab, vol. 3, no. 2, pp. 97-107, 1977.

[4] A. I. Vinik, M. L. Nevoret, C. Casellini, and H. Parson, "Diabetic neuropathy," Endocrinology and Metabolism Clinics of North America, vol. 42, no. 4, pp. 747-787, 2013.

[5] A. Izenberg, B. A. Perkins, and V. Bril, "Diabetic neuropathies," Seminars in Neurology, vol. 35, no. 4, pp. 424-430, 2015.

[6] A. P. Mizisin, "Mechanisms of diabetic neuropathy: Schwann cells," Handbook of Clinical Neurology, vol. 126, pp. 401-428, 2014.

[7] S. Sharghi-Namini, M. Turmaine, C. Meier et al., "The structural and functional integrity of peripheral nerves depends on the glial-derived signal desert hedgehog," Journal of Neuroscience, vol. 26, no. 23, pp. 6364-6376, 2006.

[8] L. Eckersley, "Role of the Schwann cell in diabetic neuropathy," International Review of Neurobiology, vol. 50, pp. 293321, 2002.

[9] K. Naruse, "Schwann Cells as Crucial Players in Diabetic Neuropathy," Advances in Experimental Medicine and Biology, vol. 1190, pp. 345-356, 2019.

[10] N. P. Goncalves, C. B. Vaegter, H. Andersen, L. Ostergaard, N. A. Calcutt, and T. S. Jensen, "Schwann cell interactions with axons and microvessels in diabetic neuropathy," Nature Reviews Neurology, vol. 13, no. 3, pp. 135-147, 2017.

[11] Y. P. Liu, S. J. Shao, and H. D. Guo, "Schwann cells apoptosis is induced by high glucose in diabetic peripheral neuropathy," Life Sciences, vol. 248, article 117459, 2020.

[12] C. De Gregorio, D. Contador, M. Campero, M. Ezquer, and F. Ezquer, "Characterization of diabetic neuropathy progression in a mouse model of type 2 diabetes mellitus," Biology Open, vol. 7, no. 9, 2018.

[13] O. Erbas, D. Taşkıran, F. Oltulu et al., "Oxytocin provides protection against diabetic polyneuropathy in rats," Neurological Research, vol. 39, no. 1, pp. 45-53, 2017.

[14] K. A. Lee, N. Y. Lee, T. S. Park, and H. Y. Jin, “Comparison of peripheral nerve protection between insulin-based glucose control and alpha lipoic acid (ALA) in the streptozotocin
(STZ)-induced diabetic rat," Endocrine, vol. 61, no. 1, pp. 58-67, 2018.

[15] K. Li, X. Shi, M. Luo et al., "Taurine protects against myelin damage of sciatic nerve in diabetic peripheral neuropathy rats by controlling apoptosis of schwann cells via NGF/Akt/GSK3 $\beta$ pathway," Experimental Cell Research, vol. 383, no. 2, article 111557, 2019.

[16] Y. L. Tiong, K. Y. Ng, R. Y. Koh, G. Ponnudurai, and S. M. Chye, "Melatonin Prevents Oxidative Stress-Induced Mitochondrial Dysfunction and Apoptosis in High GlucoseTreated Schwann Cells via Upregulation of $\mathrm{Bcl} 2, \mathrm{NF}-\kappa \mathrm{B}$, mTOR, Wnt Signalling Pathways," Antioxidants, vol. 8, no. 7, 2019.

[17] P. Y. Chao, J. A. Lin, J. C. Ye et al., "Attenuation of Oxidative Stress-Induced Cell Apoptosis in Schwann RSC96 Cells by Ocimum Gratissimum Aqueous Extract," International Journal of Medical Sciences, vol. 14, no. 8, pp. 764-771, 2017.

[18] M. Fidanboylu, L. A. Griffiths, and S. J. L. Flatters, "Global inhibition of reactive oxygen species (ROS) inhibits paclitaxel-induced painful peripheral neuropathy," PLoS ONE, M. L. Baccei, Ed., vol. 6, no. 9, article e25212, 2011.

[19] W. Kallenborn-Gerhardt, K. Schröder, D. Del Turco et al., "NADPH Oxidase-4 Maintains Neuropathic Pain after Peripheral Nerve Injury," Journal of Neuroscience, vol. 32, no. 30, pp. 10136-10145, 2012.

[20] B. Uttara, A. V. Singh, P. Zamboni, and R. T. Mahajan, “Oxidative Stress and Neurodegenerative Diseases: A Review of Upstream and Downstream Antioxidant Therapeutic Options," Current Neuropharmacology, vol. 7, no. 1, pp. 65-74, 2009.

[21] L. Q. Sun, B. Xue, X. J. Li et al., "Inhibitory effects of Salvianolic acid B on apoptosis of Schwann cells and its mechanism induced by intermittent high glucose," Life Sciences, vol. 90, no. 3-4, pp. 99-108, 2012.

[22] L. Q. Sun, Y. Y. Chen, X. Wang et al., "The protective effect of alpha lipoic acid on Schwann cells exposed to constant or intermittent high glucose," Biochemical Pharmacology, vol. 84, no. 7, pp. 961-973, 2012.

[23] S. H. Choi, Y. M. Kim, J. M. Lee, and S. G. Kim, “Antioxidant and mitochondrial protective effects of oxidized metabolites of oltipraz," Expert Opinion on Drug Metabolism \& Toxicology, vol. 6, no. 2, pp. 213-224, 2010.

[24] M. E. Killeen, J. A. Englert, D. B. Stolz et al., "The Phase Enzyme Inducers Ethacrynic Acid, DL-Sulforaphane, and Oltipraz Inhibit Lipopolysaccharide-Induced High-Mobility Group Box 1 Secretion by RAW 264.7 Cells," Journal of Pharmacology and Experimental Therapeutics, vol. 316, no. 3, pp. 1070-1079, 2006.

[25] S. Sajadimajd and M. Khazaei, "Oxidative Stress and Cancer: The Role of Nrf2," Current Cancer Drug Targets, vol. 18, no. 6, pp. 538-557, 2018.

[26] H. Zhang, K. J. A. Davies, and H. J. Forman, "Oxidative stress response and Nrf2 signaling in aging," Free Radical Biology and Medicine, vol. 88, no. Part B, pp. 314-336, 2015.

[27] I. Bellezza, I. Giambanco, A. Minelli, and R. Donato, "Nrf2Keap1 signaling in oxidative and reductive stress," Biochimica et Biophysica Acta (BBA) - Molecular Cell Research, vol. 1865, no. 5, pp. 721-733, 2018.

[28] A. Noorafshan, S. Kardeh, S. Ashkani-Esfahani, M. R. Namazi, and E. Saleh, "The Effects of Oltipraz on Tissue Regeneration in the Process of Wound Healing: A Stereological Study," 
Bulletin of Emergency And Trauma, vol. 2, no. 4, pp. 161-165, 2014.

[29] A. F. Díaz, S. Polo, N. Gallardo, S. Leánez, and O. Pol, “Analgesic and Antidepressant Effects of Oltipraz on Neuropathic Pain in Mice by Modulating Microglial Activation," Journal of Clinical Medicine, vol. 8, no. 6, 2019.

[30] Y. Tang, M. Guo, X. Y. Ma, W. P. Sun, M. H. Hao, and H. Y. Zhu, "Oltipraz attenuates the progression of heart failure in rats through inhibiting oxidative stress and inflammatory response," European Review for Medical and Pharmacological Sciences, vol. 22, no. 24, pp. 8918-8923, 2018.

[31] H. Yu, T. Lin, W. Chen et al., "Size and temporal-dependent efficacy of oltipraz-loaded PLGA nanoparticles for treatment of acute kidney injury and fibrosis," Biomaterials, vol. 219, article 119368, 2019.

[32] Y. Chang, F. Wang, Y. Yang et al., “Acetaminophen-induced hepatocyte injury: C2-ceramide and oltipraz intervention, hepatocyte nuclear factor 1 and glutathione S -transferase A1 changes," Journal of Applied Toxicology, vol. 39, no. 12, pp. 1640-1650, 2019.

[33] E. L. Feldman, K. A. Nave, T. S. Jensen, and D. L. H. Bennett, "New Horizons in Diabetic Neuropathy: Mechanisms, Bioenergetics, and Pain," Neuron, vol. 93, no. 6, pp. 1296-1313, 2017.

[34] M. Zhou, N. Chen, L. He et al., "Oxcarbazepine for neuropathic pain," Cochrane Database of Systematic Reviews, vol. 12, 2017.

[35] L. C. Rolim, E. M. da Silva, R. L. Flumignan, M. M. Abreu, S. A. Dib, and Cochrane Neuromuscular Group, "Acetyl-L-carnitine for the treatment of diabetic peripheral neuropathy," Cochrane Database of Systematic Reviews, vol. 6, 2019.

[36] F. J. Bock and S. W. G. Tait, "Mitochondria as multifaceted regulators of cell death," Nature Reviews Molecular Cell Biology, vol. 21, no. 2, pp. 85-100, 2020. 